\title{
Variabilidade espacial da chuva durante 0 experimento LBA/TRMM 1999 na Amazônia
}

\author{
Gilberto FISCH${ }^{1}$, Iria F. VENDRAME ${ }^{2}$, Paulo Cesar de Melo HANAOKA ${ }^{2}$ \\ RESUMO \\ O objetivo desta pesquisa foi o de estudar a variabilidade espacial de chuvas convectivas na Amazônia, durante o experimento \\ LBA/TRMM em 1999. Um conjunto de dados consistindo de 37 pluviômetros (divididos em 4 subconjuntos e com distância \\ máxima entre eles de $50 \mathrm{~km}$ ) foi utilizado, sendo estas medidas de pluviometria de meados de Dezembro de 1998 ao final \\ de Fevereiro de 1999, que é o pico da estação chuvosa em Rondônia (sudoeste da Amazônia). A metodologia de correlação \\ interestações (baseado em probabilidade condicional) e assumindo uma distribuição estatística log-normal bivariada foi aplicada \\ aos dados de precipitação diária e os resultados mostraram que chuvas que ocorrem em uma distância inferior a $1 \mathrm{~km}$ de raio \\ têm um alto valor de correlação (variando de 0,7 a 0,9 ) representando a validação de uma medida pontual de chuva. A curva \\ ajustada da variação do coeficiente de correlação $(\rho)$ versus distância $(\mathrm{d}$ em km) foi: $\rho=0,72-0,15 \ln (\mathrm{d})$.
}

PALAVRAS-CHAVE: Metodologia interestação, Coeficiente de correlação, Chuvas convectivas, Pluviômetros, Sistemas convectivos de meso-escala

\section{Rainfall space variability during the LBA/TRMM experiment 1999 in Amazônia}

\section{ABSTRACT}

The purpose of this work was to study the space variability of the convective rainfall in Amazonia during the LBA/TRMM experiment 1999. A dataset of 37 rain gauges (split in 4 networks and geographically located with a maximum distance amongst them around $50 \mathrm{~km}$ ) has been used to provide rainfall measurements for the period from mid Dec 1998 until end of Feb 1999, which is the peak of the rainy season at Rondonia (southwest of Amazonia). The methodology of interstation correlation (based on conditional probability and assuming a bivariate mixed lognormal distribution) have been applied to computed daily rainfall and the results had shown that rainfalls occurring within a $1 \mathrm{~km}$ of radius have a high correlation coefficient (ranging from 0.7 up to 0.9 ) representing the validation of a point rainfall measurement. The adjusted curve of the correlation coefficient $(\rho)$ vs distance $(\mathrm{d}$ in $\mathrm{km})$ was: $\rho=0.72-0.15 \ln (\mathrm{d})$.

KEYWORDS: Interstation methodology, Correlation coefficient, Convective rainfall, Raingauges, Mesoscale convective system.

1 Instituto de Aeronáutica e Espaço (IAE). Centro Técnico Aeroespacial, Instituto de Aeronáutica e Espaço, Divisão de Ciências Atmosféricas. Praça Marechal Eduardo Gomes, 50 Vila das Acácias. CEP 12228-904 - São Jose dos Campos, SP - Brasil. Telefone: (12) 39474565 Fax: (12) 39474550. e-mail: gfisch@iae.cta.br

2 Instituto Tecnológico da Aeronáutica (ITA). 


\section{INTRODUÇÃO}

A Floresta Amazônica é um dos mais importantes ecossistemas do planeta, sendo que sua biodiversidade e sua importância no equilíbrio do planeta estão sendo cada vez mais discutidas. A Amazônia é também a maior bacia hidrográfica do mundo, com cerca de cinco milhōes de quilômetros quadrados e influi nos ciclos biogeoquímicos e hidrológicos, com interações no clima do mundo inteiro. Sendo uma floresta equatorial de clima quente e úmido, a Floresta Amazônica possui uma alta pluviosidade (valores médios de precipitação anual entre 2.000 a $2.200 \mathrm{~mm}$ ) associada a uma forte atividade convectiva, com períodos longos e intensos de chuvas durante o ano. A precipitação no pico do período chuvoso normalmente excede o valor de 250 $\mathrm{mm} / \mathrm{mês}$. Durante a estação seca, com a retração do sistema de larga-escala de convecção (denominado de Alta da Bolívia) para a região NW da América do Sul (Horel et al., 1989), a parte sudoeste da Amazônia (no qual inclui-se o estado de Rondônia) apresenta uma estação seca bem definida, com totais mensais de precipitação inferior a $50 \mathrm{~mm} / \mathrm{mês}$ (Ferreira da Costa et al., 1998).

As chuvas convectivas são características das regiōes equatoriais. Uma vez que os ventos são fracos, a movimentação do ar ocorre basicamente na vertical (Tucci, 1997), produzindo processos de convecção, nuvens e chuvas. Este processo é tipicamente local (escala de variação inferior a 1 $\mathrm{km}$ ) e de curta duração (tempo menor que 1 hora). Outra característica importante associada a este processo é a questão da intermitência da ocorrência de chuvas. É na Amazônia que ocorrem as principais atividades convectivas no planeta e que pode ter uma influência grande no clima de outras localidades, devido ao transporte horizontal de energia e vapor d'água. Atualmente, por exemplo, estuda-se de que forma o transporte de umidade atmosférica da Amazônia pode influenciar no regime pluviométrico da região da Bacia do Rio da Prata, distante mais de $3.000 \mathrm{~km}$ (Vera et al., 2006).

As informaçōes sobre a precipitação na Amazônia, embora seja o elemento climático com o maior número de observações disponíveis, ainda são escassas e, sobretudo, concentram-se nas cidades, ou ao longo dos meios de transporte (rios e rodovias). Molion \& Dallarosa (1990), por exemplo, discutem alguns dos erros originados dos estudos do regime pluviométrico na Amazônia que consideram dados, em sua grande maioria, ao longo dos rios. Mais recentemente, Tota et al. (2000) e Marengo et al. (2004) analisaram o mesmo conjunto de dados deste trabalho, com o intuito de analisar as ocorrências de chuvas em um único ponto (Tota et al., 2000) ou abrangendo o estado inteiro (Marengo et al., 2004). Porém estes autores não analisaram como ocorrem as chuvas convectivas e suas interrelações espaço-temporais. Estas relaçôes são importantes para se extrapolar medidas pontuais para representar uma determinada área em posteriores aplicaçôes hidrológicas, tais como estimativa do volume de água precipitado sobre uma bacia hidrográfica durante uma tormenta. A análise de freqüência de precipitaçōes sobre uma área não é ainda tão desenvolvida quanto à análise de precipitação num ponto. $\mathrm{Na}$ ausência de informação sobre a verdadeira distribuição de probabilidade da precipitação na área, as estimativas de precipitação no ponto são usualmente estendidas para se obter uma precipitação média sobre uma dada área. Devido à escassez de estudos regionais sobre a distribuição espacial da precipitação, esta redução tem sido realizada utilizando relaçōes obtidas pelo Weather Bureau (1946), com base numa rede de pluviógrafos cobrindo áreas de 100 a 400 milhas quadradas, localizadas na sua maioria a leste do rio Mississipi (EUA), sendo que o número médio de postos em cada rede foi de 6 e as séries variaram de 7 a 15 anos. A Figura 1 apresenta as curvas de variação espacial da altura precipitada para chuvas de diferentes durações obtidas pelo Weather Bureau (1946).

Segundo Krajewski \& Ciach (2003), mesmo com o aumento do conhecimento da estrutura espacial em escalas de 10 a 1000 km nas últimas décadas, a completa compreensão da estrutura da chuva em escala pequena (dezenas de metros a poucos quilômetros) ainda é deficiente, principalmente a falta de dados observacionais com esta resolução. A magnitude da variabilidade espaço-temporal pode ser devida a variabilidade natural das chuvas e/ou erros instrumentais. Krajewski \& Ciach (2003) mostram ainda como a variabilidade espacialtemporal variam para pluviômetros separados entre 1 a 1100 $\mathrm{m}$, para chuvas entre 5 e $60 \mathrm{~min}$, em 5 locais de diferentes regimes climáticos (incluindo a Amazônia).

Este trabalho tem por finalidade analisar dados de precipitação com alta resolução espacial obtidos pelo experimento LBA/TRMM 1999 (maiores informações em

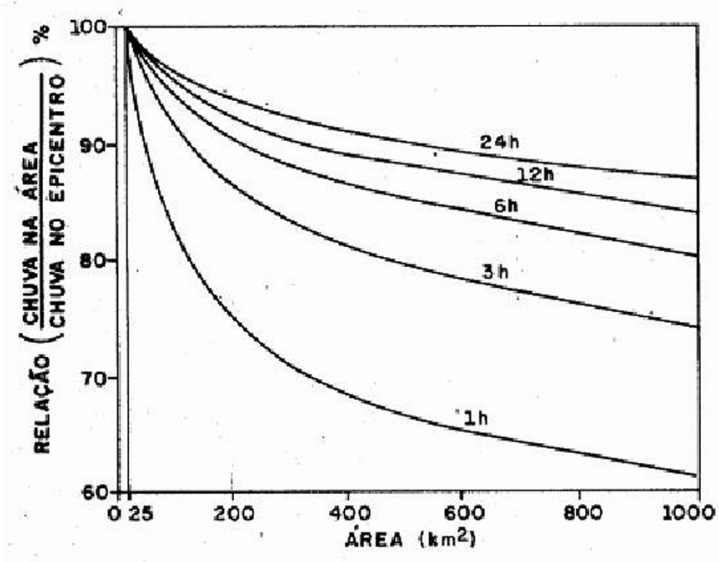

Figura 1 - Relação entre a chuva média na área e a chuva máxima no epicentro tormentas.

Fonte: Weather Bureau, (1946). 
Silva Dias et al., 2002 ou em http://www.master.iag.usp.br/ lba) e determinar como ocorre a distribuição espacial das chuvas, ajudando a caracterizar e compreender os eventos de precipitação convectiva que ocorrem na região Amazônica.

\section{MATERIAL E MÉTODOS}

O estudo foi realizado com base em dados de pluviometria coletados durante o experimento LBA/TRMM 1999 (Tota et al., 2000; Marengo et al., 2004). Foram instalados 37 pluviômetros do tipo caçambas basculantes ("tipping bucket") em quatro grupos ("networks") de pluviômetros, sendo 14 pluviômetros instalados no "network 1", 13 pluviômetro no "network 2", 5 pluviômetros no "network 3" e 5 pluviômetros no "network 4", espalhados em diversas localidades de Rondônia, centrados na região de Ji-Paraná (RO). A distância máxima entre os pluviômetros foi de $50 \mathrm{~km}$. A Figura 2 apresenta de forma sintética o posicionamento destes "networks" em RO, bem como de outros pontos de medidas de precipitação durante o experimento LBA/TRMM, mas que não foram utilizados neste trabalho (veja uma análise detalhada do regime pluviométrico em Marengo et al., 2004).

O pluviômetro consiste de um receptáculo onde a água da chuva é coletada por uma caçamba dividida em dois compartimentos, sendo que cada vez que o nível é completado, a caçamba gira, descarrega a água e é feita uma contagem. Como é conhecida a altura de chuva que faz com que a báscula gire (normalmente $0,25 \mathrm{~mm}$ ), e uma vez que é registrada a contagem de giros, pode-se calcular, dessa forma, o volume de água precipitado. No pluviômetro existe um sistema de aquisição de dados ("data-logger"), o qual registra o horário em que ocorrem as basculadas. As informações de precipitação ficam desta forma, armazenadas no sistema de registro de dados até que sejam retiradas digitalmente as informaçôes do pluviômetro.

O período de coleta de dados foi de 23/12/1998 a 28/02/1999, que caracteriza a estação chuvosa da região.

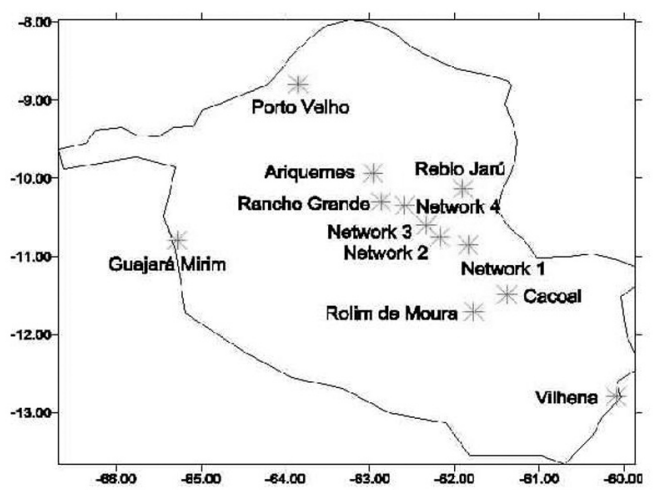

Figura 2 - Mapa do estado de Rondônia com a localização geográfica dos "networks" 1 a 4 durante 0 experimento LBA/TRMM 1999.
Os instrumentos foram calibrados antes da coleta de dados. Embora este período seja relativamente pequeno (dois meses), o total de precipitação foi alto (superior a $500 \mathrm{~mm}$ ), com muitos eventos de chuvas (basicamente originadas de processos convectivos). Neste período também não ocorreram anomalias nos campos de precipitação e de temperatura da região, apresentando condiçôes meteorológicas típicas da região para essa época do ano. Com relação ao "controle de qualidade CQ" dos dados, pequenas distorçôes e erros absurdos nos dados foram corrigidos, pois foi notado que se tratava de erros de dados. Alguns pluviômetros foram eliminados da análise, tais como os de número 3, 5, 10, 14 (todos do "network 2"), 24 ("network 3") e 33 ("network 4"), uma vez que os mesmos possuíam falhas óbvias e longas de dados.

Para analisar e caracterizar a distribuição espaço (variabilidade) da chuva convectiva utilizou-se uma metodologia que se baseia na correlação entre medidas de dois pluviômetros, bem como a distância que os separa. Esta metodologia foi proposta por Habib et al. (2001) para, a partir de dados de chuva convectiva ocorridas na Flórida durante o verão de 1998 (Experimento TEFLUN-B), calibrar as medidas efetuadas por radar meteorológico e validação dos produtos oriundos do satélite Tropical Rain Measuring Mission TRMM. Ressalta-se de que os dados utilizados nesta pesquisa também são de origem de um experimento envolvendo o satélite TRMM (Experimento LBA/TRMM 1999).

$O$ coeficiente de correlação da população $\rho(X, Y)$ é normalmente definido por:

$$
\rho(X, Y)=\frac{E(X Y)-E(X) \cdot E(Y)}{\sqrt{\operatorname{Var}(X) \cdot \operatorname{Var}(Y)}}
$$

no qual os operadores estatísticos $\mathrm{E}($ ) e $\operatorname{Var}($ ) representam o valor esperado e a variância da amostra e que podem ser estimados por valores médios e desvios-padrão. Esse coeficiente é uma medida da dependência linear entre pares de variáveis aleatórias. No caso da aplicação em chuvas, esta variável é utilizada para analisar o ajuste entre 2 tipos de medidas de chuvas, tais como pluviômetro e radar meteorológico. Entretanto, caso as variáveis em questão não possuam uma distribuição normal, este indicador possui um alto viés e é ineficiente (Krajewski \& Ciach, 2003). Este viés pode ser corrigido conforme o procedimento descrito abaixo.

Habib et al. (2001) cita diversos modelos possíveis de ajuste de distribuição estatística, tais como a distribuição gama, hiperbólica e lognormal. Dentre estas e considerando que não existe uma aceitação única da comunidade científica sobre a distribuição adequada, escolheu-se a distribuição lognormal, conforme estudo realizado por Shimizu (1993). 
O modelo adotado (lognormal) leva em conta a característica intermitente da chuva. Shimizu (1993) desenvolveu um modelo estatístico com distribuição lognormal bivariada, com a finalidade de analisar a distribuição espacial da chuva na cidade de Tokyo, a qual considera que os dados de chuva podem ser divididos em quatro casos distintos:

1) Precipitação nula em ambos os pluviômetros: $(0 ; 0)$

2) Precipitação nula apenas no primeiro pluviômetro: $\left(0 ; y^{*}\right)$

3) Precipitação nula apenas no segundo pluviômetro: $\left(x^{*} ; 0\right)$

Precipitação não-nula em ambos os pluviômetros: $(x, y)$

Dessa forma, dada uma amostra de precipitação com tamanho $N$, os quatro casos terão tamanhos $n_{0}, n_{1}, n_{2}$ e $n_{3}$ onde $N=n_{0}+n_{1}+n_{2}+n_{3}$. A distribuição dada pode ser descrita completamente com os seguintes parâmetros:

a) $\delta_{0}, \delta_{1}, \delta_{2}, \delta_{3}$ :Probabilidade de ocorrência de cada caso descrito;

b) $\mu_{1}{ }^{*}, \mu_{2}{ }^{*}, \sigma_{1}{ }^{*}, \sigma_{2}{ }^{*}$ : Média e desvio padrão para os casos e , respectivamente;

c) $\mu_{1}, \mu_{2}, \sigma_{1}, \sigma_{2}, \rho_{N}$ : Média, desvio padrão e coeficiente de correlação para o caso $n_{3}$.

A média e o desvio padrão da população, seguindo a formulação de Shimizu (1983), podem ser descritas por:

$$
\begin{aligned}
& \mathrm{E}(\mathrm{X})=\delta_{1} \exp \left[\mu_{1}^{*}+\left(\sigma_{1}^{* 2} / 2\right)\right]+\delta_{3} \exp \left[\mu_{1}+\left(\sigma_{1}^{2} / 2\right)\right] \\
& \mathrm{E}(\mathrm{Y})=\delta_{2} \exp \left[\mu_{2}^{*}+\left(\sigma_{2}^{* 2} / 2\right)\right]+\delta_{3} \exp \left[\mu_{2}+\left(\sigma_{2}^{2} / 2\right)\right] \\
& \mathrm{E}(\mathrm{XY})=\delta_{3} \exp \left[\mu_{1}+\mu_{2}+\left(\sigma_{1}^{2}+\sigma_{2}^{2}+2 \sigma_{1} \sigma_{2} \rho_{N}\right) / 2\right]
\end{aligned}
$$

$\operatorname{VAR}(\mathrm{X})=$

$\delta_{1} \exp \left[\left(2 \mu_{1}^{*}+2 \sigma_{1}^{\circ 2}\right)\right]+\delta_{3} \exp \left(2 \mu_{1}+2 \sigma_{1}^{2}\right)-\left\{\delta_{1} \exp \left[\mu_{1}^{*}+\left(\sigma_{1}^{* 2} / 2\right)\right]+\delta_{3} \exp \left[\mu_{1}+\left(\sigma_{1}^{2} / 2\right)\right]^{2}\right.$

$\operatorname{VAR}(\mathrm{Y})=$

$\delta_{2} \exp \left[\left(2 \mu_{2}^{*}+2 \sigma_{2}^{\prime 2}\right)\right]+\delta_{3} \exp \left(2 \mu_{2}+2 \sigma_{2}^{2}\right)-\left\{\delta_{1} \exp \left[\mu_{2}^{*}+\left(\sigma_{2}^{*} / 2\right)\right]+\delta_{3} \exp \left[\mu_{2}+\left(\sigma_{2}^{2} / 2\right)\right]^{2}\right.$

Substituindo estas expressões acima na equação (1), é possível calcular o coeficiente de correlação da população, a partir de uma distribuição lognormal bivariada.

Considerando o caso em que (Caso M1 de Shimizu, 1983) e utilizando-se o método da máxima verossimilhança, é possível calcular o valor do coeficiente de correlação da precipitação entre dois pluviômetros.

Foi considerado o caso M1 de Shimizu (1983), pois as análises realizadas por Habib et al. (2001) para o conjunto de dados de TEFLUN-B geraram valores de médias e desvio padrōes que são diferentes entre si. Os dados de chuva do TEFLUN-B são oriundos de chuvas convectivas na Flórida, que são semelhantes com as chuvas que ocorrem na Amazônia, em termos de gênese, formação e aspectos.

\section{RESULTADOS E DISCUSSÃO}

As informações originais armazenadas nos pluviômetros instalados no campo foram do início e final de cada chuva, além da quantidade de água precipitada. Estas informaçôes foram convertidas para precipitação diária e arredondadas para valores inteiros (unidade em $\mathrm{mm}$ ), conforme as premissas do trabalho de Habib et al. (2001) e de Shimizu (1993). Embora tenham sido utilizados totais diário de chuvas, os eventos de precipitação foram isolados, ou seja, as chuvas ocorreram apenas uma vez ao dia, caracterizando eventos convectivos. Além disso, embora o período de coleta de dados seja relativamente pequeno (2 meses), o total de precipitação acumulado foi alto, dentro da climatologia observada.

A análise da precipitação acumulada média no estado de Rondônia nos meses de Janeiro e Fevereiro revela uma parte das características da época chuvosa. Segundo Ferreira da Costa et al. (1998), no período de 1982 até 1996, a precipitação acumulada média para Janeiro e Fevereiro foi de $566 \mathrm{~mm}$. Segundo Marengo et al. (2004), a média de precipitação acumulada de 22 de Dezembro de 1998 até 28 de Fevereiro de 1998, para estes mesmos 4 "networks" foi de $516 \mathrm{~mm}$. Foi também verificada a ocorrência de períodos com altas precipitaçóes centrados nos dias 23 de Janeiro, 15-18 de Fevereiro e 24-25 de Fevereiro. Nestes últimos dois casos, provavelmente as fortes precipitaçōes foram decorrentes de sistemas de mesoescala que passaram pela região (Tota et al., 2000).

Com a aplicação do método proposto foi possível obter a correlação entre todos os pluviômetros analisados. A distância entre pluviômetros foi calculada empregando-se as coordenadas geográficas de cada um. A Tabela 1 apresenta a distância (diagonal inferior) e os valores de correlação (diagonal superior) entre os pares de pluviômetros. Estas informaçôes permitiram construir um gráfico da correlação entre os pluviômetros em função da distância que os separa (Figura 3) para todos os pares de valores analisados. Optou-se pela adoção de um ajuste logaritmico (método dos mínimos quadrados) para a escolha da melhor curva que se adapta aos dados, a saber:

$$
\rho=0,7169-0,1452 \ln (\mathbf{d}) .
$$

onde, $\rho$ é a correlação (adimensional) e $\boldsymbol{d}$ é a distância (em $\mathrm{km}$ ) entre dois pluviômetros quaisquer. Através desta relação, pode-se calcular que a correlação varia de um valor de 0,86 
Tabela 1 - Valores de distância (diagonal inferior) e correlação (diagonal superior) entre os pluviômetros

\begin{tabular}{|c|c|c|c|c|c|c|c|c|c|c|c|c|c|c|c|}
\hline & 1 & 2 & 4 & 6 & 7 & 8 & 9 & 11 & 12 & 13 & 15 & 16 & 17 & 18 & 19 \\
\hline 1 & & 0,94 & 0,94 & 0,67 & 0,66 & 0,67 & 0,67 & 0,62 & 0,62 & 0,71 & 0,41 & 0,39 & 0,35 & 0,42 & 0,44 \\
\hline 2 & 0,8 & & 0,99 & 0,75 & 0,74 & 0,74 & 0,74 & 0,69 & 0,70 & 0,70 & 0,35 & 0,38 & 0,30 & 0,41 & 0,45 \\
\hline 4 & 0,6 & 0,6 & & 0,75 & 0,74 & 0,75 & 0,74 & 0,64 & 0,70 & 0,70 & 0,36 & 0,39 & 0,30 & 0,42 & 0,45 \\
\hline 6 & 1,3 & 0,6 & 0,8 & & 0,99 & 1,00 & 0,99 & 0,74 & 0,85 & 0,66 & 0,36 & 0,43 & 0,39 & 0,43 & 0,46 \\
\hline 7 & 1,3 & 0,6 & 0,8 & 0,0 & & 1,00 & 0,99 & 0,74 & 0,85 & 0,63 & 0,38 & 0,42 & 0,37 & 0,45 & 0,46 \\
\hline 8 & 1,3 & 0,6 & 0,8 & 0,0 & 0,0 & & 0,99 & 0,74 & 0,87 & 0,64 & 0,37 & 0,42 & 0,36 & 0,44 & 0,46 \\
\hline 9 & 1,3 & 0,6 & 0,8 & 0,0 & 0,0 & 0,0 & & 0,74 & 0,86 & 0,68 & 0,39 & 0,45 & 0,42 & 0,42 & 0,48 \\
\hline 11 & 1,6 & 0,8 & 1,3 & 0,6 & 0,6 & 0,6 & 0,6 & & 0,67 & 0,59 & 0,34 & 0,39 & 0,33 & 0,33 & 0,41 \\
\hline 13 & 1,2 & 1,2 & 1,6 & 1,8 & 1,8 & 1,8 & 1,8 & 1,7 & 1,7 & & 0,39 & 0,37 & 0,30 & 0,44 & 0,55 \\
\hline 15 & 17,0 & 16,6 & 17,1 & 16,8 & 16,8 & 16,8 & 16,8 & 16,3 & 16,3 & 15,7 & & 0,61 & 0,60 & 0,58 & 0,55 \\
\hline 16 & 18,7 & 18,4 & 18,9 & 18,6 & 18,6 & 18,6 & 18,6 & 18,0 & 18,1 & 17,5 & 1,8 & & 0,94 & 0,60 & 0,65 \\
\hline 17 & 18,7 & 18,4 & 18,9 & 18,6 & 18,6 & 18,6 & 18,6 & 18,0 & 18,1 & 17,5 & 1,8 & 0,0 & & 0,49 & 0,66 \\
\hline 18 & 20,6 & 20,3 & 20,8 & 20,5 & 20,5 & 20,5 & 20,5 & 20,0 & 20,0 & 19,4 & 3,7 & 2,0 & 2,0 & & 0,55 \\
\hline 19 & 20,0 & 19,6 & 20,2 & 19,8 & 19,8 & 19,8 & 19,8 & 19,2 & 19,3 & 18,8 & 3,4 & 2,0 & 2,0 & 2,2 & \\
\hline 20 & 19,9 & 19,6 & 20,1 & 19,8 & 19,8 & 19,8 & 19,8 & 19,3 & 19,3 & 18,7 & 3,0 & 1,2 & 1,2 & 0,8 & 1,8 \\
\hline 21 & 19,9 & 19,6 & 20,1 & 19,8 & 19,8 & 19,8 & 19,8 & 19,3 & 19,3 & 18,7 & 3,0 & 1,2 & 1,2 & 0,8 & 1,8 \\
\hline 23 & 19,9 & 19,6 & 20,1 & 19,8 & 19,8 & 19,8 & 19,8 & 19,3 & 19,3 & 18,7 & 3,0 & 1,2 & 1,2 & 0,8 & 1,8 \\
\hline 25 & 19,5 & 19,1 & 19,6 & 19,2 & 19,2 & 19,2 & 19,2 & 18,7 & 18,7 & 18,3 & 2,8 & 1,6 & 1,6 & 2,3 & 0,6 \\
\hline 26 & 19,5 & 19,1 & 19,6 & 19,2 & 19,2 & 19,2 & 19,2 & 18,7 & 18,7 & 18,3 & 2,8 & 1,6 & 1,6 & 2,3 & 0,6 \\
\hline 27 & 19,5 & 19,1 & 19,6 & 19,2 & 19,2 & 19,2 & 19,2 & 18,7 & 18,7 & 18,3 & 2,8 & 1,6 & 1,6 & 2,3 & 0,6 \\
\hline 28 & 32,2 & 32,0 & 32,5 & 32,3 & 32,3 & 32,3 & 32,3 & 31,8 & 31,8 & 31,0 & 15,9 & 14,3 & 14,3 & 12,3 & 13,9 \\
\hline 29 & 31,7 & 31,5 & 32,0 & 31,8 & 31,8 & 31,8 & 31,8 & 31,3 & 31,3 & 30,5 & 15,4 & 13,8 & 13,8 & 11,9 & 13,5 \\
\hline 30 & 31,5 & 31,2 & 31,7 & 31,5 & 31,5 & 31,5 & 31,5 & 31,0 & 31,0 & 30,2 & 15,1 & 13,5 & 13,5 & 11,5 & 13,1 \\
\hline 31 & 31,7 & 31,5 & 32,0 & 31,8 & 31,8 & 31,8 & 31,8 & 31,3 & 31,3 & 30,5 & 15,4 & 13,8 & 13,8 & 11,9 & 13,5 \\
\hline 32 & 31,5 & 31,2 & 31,7 & 31,5 & 31,5 & 31,5 & 31,5 & 31,0 & 31,0 & 30,2 & 15,1 & 13,5 & 13,5 & 11,5 & 13,1 \\
\hline 34 & 49,6 & 49,5 & 49,9 & 49,8 & 49,8 & 49,8 & 49,8 & 49,6 & 49,4 & 48,4 & 34,0 & 32,4 & 32,4 & 30,5 & 32,1 \\
\hline 35 & 50,2 & 50,0 & 50,5 & 50,4 & 50,4 & 50,4 & 50,4 & 49,9 & 49,9 & 49,0 & 34,4 & 32,8 & 32,8 & 30,8 & 32,4 \\
\hline 36 & 50,6 & 50,5 & 51,0 & 50,8 & 50,8 & 50,8 & 50,8 & 50,4 & 50,4 & 49,4 & 34,8 & 33,2 & 33,2 & 31,2 & 32,8 \\
\hline 37 & 49,7 & 49,6 & 50,0 & 49,9 & 49,9 & 49,9 & 49,9 & 49,5 & 49,5 & 48,5 & 34,0 & 32,4 & 32,4 & 30,4 & 32,0 \\
\hline
\end{tabular}

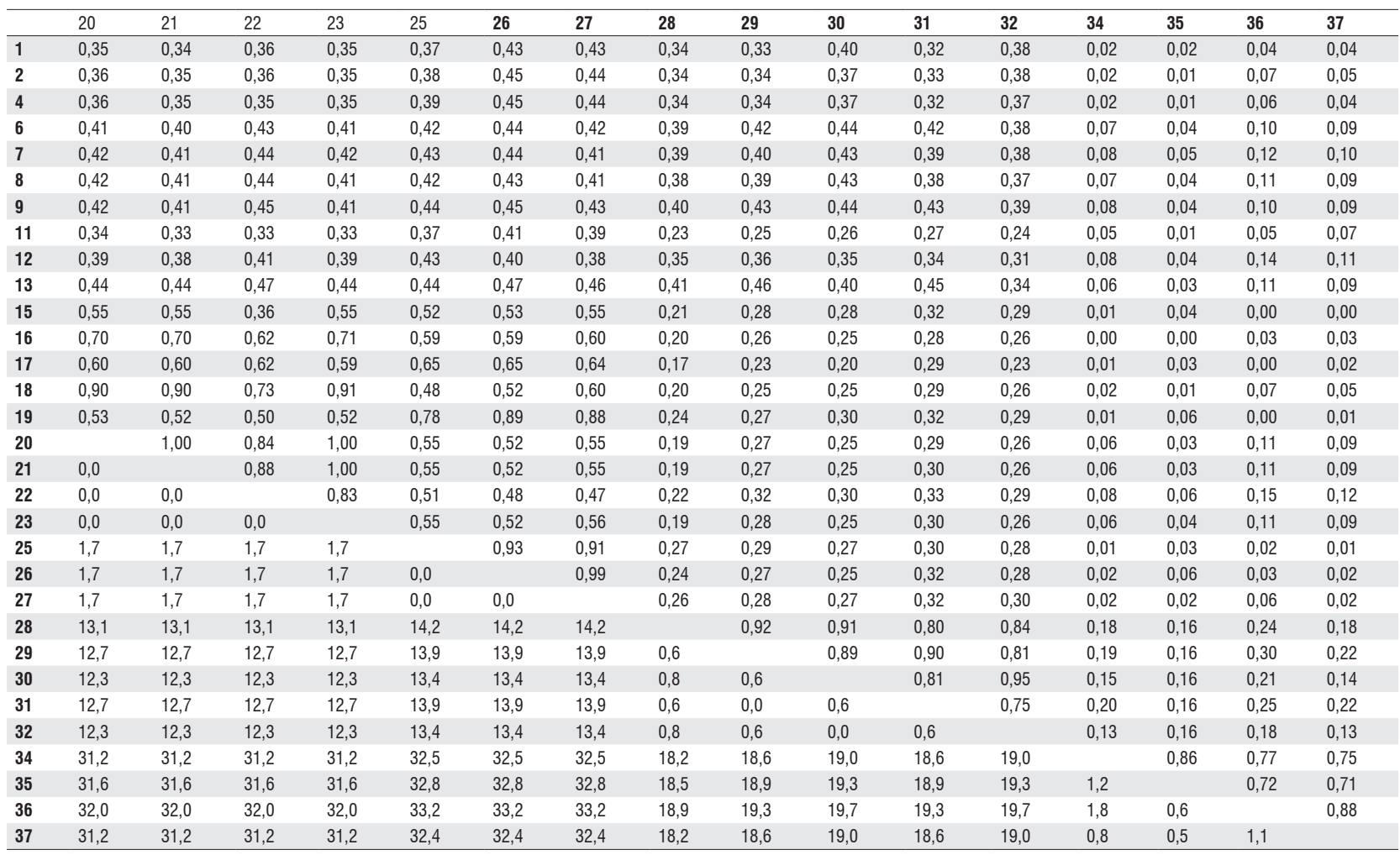


para células convectivas muito próximas (distância inferior a $0,5 \mathrm{~km}$ ) até 0,75 para uma distância de $1 \mathrm{~km}$. No caso de uma distância de $2 \mathrm{~km}$, o coeficiente de correlação é de 0,63 . Para distâncias superiores, o valor do coeficiente decai para 0,48, 0,38 e 0,15 para distâncias de 5,10 e $50 \mathrm{~km}$, respectivamente.

A seguir, visando analisar a influência de chuvas estratiformes no conjunto total de dados, realizou-se uma simulação no qual se retirou os dias onde ocorreram fenômenos de mesoescala. Segundo Silva Dias et al. (2002), a convecção local pode estar em parte associada com a topografia da região e o uso da terra (floresta ou pastagem), sendo que ocorreram, no período do experimento LBA/TRMM 1999, um total de 25 casos de sistemas de mesoescala. Os casos mais intensos foram os eventos de 15 a 18 e 24 a 25 de fevereiro, descritos em Tota et al. (2000). Contudo, os resultados desta simulação não foram alterados significativamente, o que permite dizer que a equação encontrada pode descrever bem a chuva convectiva ocorrida em Rondônia. Posteriormente, agruparam-se as correlações obtidas segundo os pluviômetros e seus respectivos "networks". Dessa forma, obtiveram-se grupos de correlaçōes da seguinte forma: 1-1, 1-2, 1-3, 1-4, 2-2, 2-3, 2-4, 3-3, 3-4. Para todas as correlaçóes pertencentes a estes grupos, tirou-se a média simples da correlação e da distância, obtendo-se a equação abaixo (3), muito parecida com a equação (2).

$$
\rho=0,7501-0,1655 \ln (\boldsymbol{d}) \text {. }
$$

Comparando-se os resultados obtidos pela aplicação da metodologia empregada nesse trabalho com aqueles obtidos pela metodologia proposta pelo Weather Bureau (1946), verifica-se que há um maior decréscimo em relação a variação na área, quando se emprega a equação 2 , do que nos valores

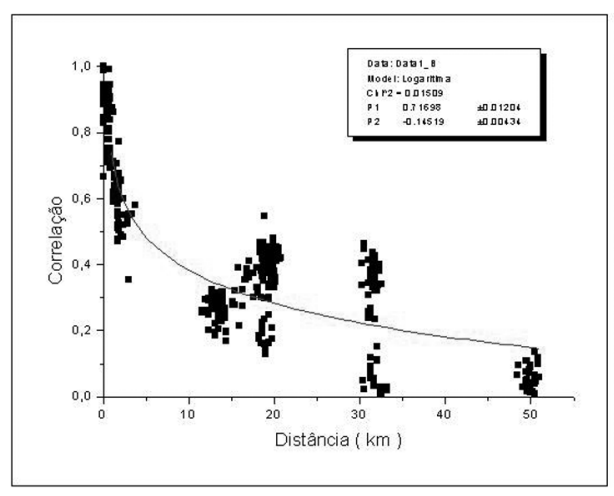

Figura 3 - Correlação (adimensional) em função da distância (em km) entre todos os pluviômetros dos vários "networks" (1 a 4) durante 0 experimento LBA/TRMM 1999. da relação (chuva na área/chuva no epicentro) obtidos pela Figura 1, para áreas de $100 \mathrm{~km}^{2}$ e $500 \mathrm{~km}^{2}$. Entretanto, deve-se ressaltar que a Figura 1 considera o epicentro ocorrendo em uma área igual a $25 \mathrm{~km}^{2}$ e chuvas com duração de 24 horas (tipicamente de origem frontal). Mas, se na Figura 1 analisar-se a curva correspondente a chuvas com duração de uma hora (tipicamente de origem convectivas), verifica-se que a relação (chuva na área/chuva no epicentro) decresce rapidamente para 0,85 para área de $100 \mathrm{~km}^{2}$ e para 0,65 para área de $500 \mathrm{~km}^{2}$, entretanto essa curva ainda fornece valores bem maiores do que aqueles obtidos pela equação 2 para pluviômetros separados por mais de $5 \mathrm{~km}$ de distância, ou seja , $\rho<0,5$.

\section{CONCLUSÕES}

A chuva na região Amazônica é predominantemente de caráter local e convectivo. Entretanto, a intermitência de sua ocorrência induz uma grande variabilidade espacial, o que por si só provoca uma incerteza da medida pontual em ser extrapolada para representar um valor de uma área (Ali et al., 2003). Nesse sentido, com a implantação do Projeto SIVAM/SIPAM e a instalação de 10 radares meteorológicos, prevê-se que será possível uma determinação melhor do regime pluviométrico da Amazônia nos próximos anos.

A estimativa da precipitação na área pode ser avaliada segundo duas consideraçôes: centro da tormenta (epicentro) ou localização fixada. Para a análise segundo a localização fixada deve-se considerar que as estaçôes medidoras estão perto do centro da tormenta, ou às vezes localizadas nas bordas da área abrangida pela tormenta, ou ainda entre o centro e a borda. As tormentas podem apresentar configurações isoiéticas complexas, mas as configuraçôes distribuem-se concentricamente em torno de um ou mais núcleos, com maiores alturas pluviométricas. Conseqüentemente, as alturas pluviométricas médias tendem a decrescer com o aumento da área sobre a qual se distribuem. Por outro lado, a distribuição isoiética tende a tornar-se uniforme com o aumento da duração da precipitação, situação que não foi objeto desse estudo, mas que deverá ser analisado posteriormente.

No caso da aplicação em chuvas, o coeficiente de correlação é utilizado para analisar o ajuste entre 2 tipos de medidas de chuvas, tais como pluviômetro e radar meteorológico. Correlação de dados de dois ou mais pluviômetros são utilizados para caracterização climática, prevenção de chuvas e também para controle de qualidade de dados. Assim sendo, seu conhecimento é importante em estudos que envolvam a chuva na região.

Os resultados obtidos neste trabalho sugerem que para pluviômetros separados por até $1 \mathrm{~km}$, a correlação permanece alta: entre 0,7 e 0,9 . Já para pluviômetros separados por mais de $5 \mathrm{~km}$ de distância, a correlação ficou entre 0,2 e 0,4. Estes valores baixos são característicos de chuvas localizadas, 
de curta duração e com céluas intensas que caracterizam as chuvas convectivas. Dessa forma, pode-se dizer que os eventos de chuva convectiva durante o experimento LBA/TRMM possuíam uma abrangência e representatividade com um raio de até $1 \mathrm{~km}$. Estes resultados podem ajudar na melhoria do entendimento de problemas relacionados com modelagem de chuva e sua inclusão em modelos de Previsão Numérica de Tempo.

\section{BIBLIOGRAFIA CITADA}

Ali, A.; Lebel; T., Amani, A. 2003. Invariance in the spatial structure of Sahelian rain fields at climatological scales. Journal of Hydrometeorology, 4(6): 996 - 1011.

Ferreira da Costa, R.; Feitosa, J.R.P.; Fisch, G.; Souza; S.S.; Nobre, C.A. 1998. Variabilidade diária da precipitação em regiōes de floresta e pastagem na Amazônia. Acta Amazônica, 28(4): 395-408.

Habib, E.; Krajewski, W. F.; Ciach, G. J. 2001. Estimation of Rainfall Interstation Correlation. Journal of Hydrometeorology, 2(3): 621-629.

Horel, J.D.; Hahmann, A.N.; Geisler, J.E. 1989. An investigation of the annual cycle of the convective activity over the tropical Americas. Journal of Climate, 2(10): 1388-1403.

Krajewski, W.F.; Ciach, G.J. 2003. An analysis of small-scale rainfall variability in different climatic regimes. Hydrological SciencesJournal des Sciences Hydrologiques, 48(2): 151-162

Marengo, J.A.; Fisch, G.; Morales, C.A.; Vendrame, I.F.; Dias, P.G.C. 2004. Diurnal variability of rainfall in southwest Amazônia during the LBA-TRMM field campaing of the Summer 1999. Acta Amazônica, 34(4): 593 - 603.
Molion, L.C.B.; Dallarosa, R.L.G. R.L.G. 1990. Pluviometria na Amazônia: são os dados confiáveis? Climanálise - Boletim de Monitoramento e Análise Climática, 5(3): 40-44.

Shimizu, K. 1993. A bivariate mixed lognormal distribution with an analysis of rainfall data. Journal of Applied Meteorology, 32(1): 161-171.

Silva Dias, M.A.F. da; Rutledge, S.; Kabat, P.; Silva Dias, P.L.da; Nobre, C.A.; Fisch, G.; Dolman, A.J.; Zipser, E.; Garstang, M.; Manzi, A.O.; Fuentes, J.D.; Rocha, H.R.; Marengo, J.A.; Plana-Fattori, A.; Sa L.D.A.; Alvala, R.C. dos Santos; Aandrea M.O.; Artaxo, P.; Gielow, R; Gatti, L. 2002. Cloud and Rain processes in a biosphere-atmosphere interaction context in the Amazon Region. Journal of Geophysical Research, 107(D20): 8072- 8090 .

Tota, J., Fisch, G., Fuentes, J., Oliveira, P.J., Garstang, M., Heitz, R., Sigler, J. 2000. Análise da variabilidade diária da precipitação em área de pastagem para a época chuvosa de 1999- Projeto TRMM/LBA. Acta Amazônica, 30(4): 629-639.

Tucci, C.E.M. 1997. Hidrologia: Ciência e Aplicação. Editora da Universidade/ABRH, Porto Alegre, RS, Brasil. 450pp

Vera, C. et al., 2006. The south American Low Level Jet Experiment (SALLJEX). Bulletin of the American Meteorological Society, 87(1): $63-77$.

Weather Bureau, 1946. Manual for Depth-area-duration Analysis of Storm Precipitation (technical paper, 1).

Recebido em 05/04/2006

Aceito em 08/06/2007 
